\title{
«Tiempos hay de acometer y tiempos de retirar»: literatura áurea y edición digital
}

\author{
Susanna Alles Torrent \\ University of Miami \\ susanna_alles@miami.edu
}

Recepción: 17/03/2017, Aceptación: 24/04/2017, Publicación: 22/12/2017

\begin{abstract}
Resumen
Cualquier estado de la cuestión actual sobre crítica textual conlleva una reflexión profunda sobre el medio digital. Muchas tradiciones nacionales han afrontado ya la transformación del modelo de edición: un modelo que implica, sobre todo, un método diferente de ejecución y una nueva presentación de los materiales. Nos encontramos pues en un momento crucial, en el que — retomando las palabras de Sancho ante la inminente participación de Don Quijote en una justa- debemos decidir si acometer o no las «nuevas» prácticas en materia de edición digital.

En el terreno de la literatura del Renacimiento y del Siglo de Oro español se han llevado a cabo proyectos de alto nivel filológico y valor digital. Este artículo pretende pasar revista a algunos de los proyectos más emblemáticos y comprometidos con las humanidades digitales, y evaluar en qué medida encajan con las guías de buenas prácticas en lo que se viene llamando edición académica digital.
\end{abstract}

Palabras clave

Edición académica digital; Siglo de Oro

\begin{abstract}
"Tiempos hay de acometer y tiempos de retirar": Golden Age literature and digital editing

Any state of the art on textual criticism entails a deep reflection on the digital medium. Many national traditions have already faced the transformation of the editorial model: a model that implies, above all, a different method of execution and a new presentation of the materials. We are currently at a crucial moment in which - calling to mind Sancho's words before the imminent participation of Don Quixote in a joust - we must decide whether or not to undertake the "new" practices within the field of digital editing. In studies of
\end{abstract}


Renaissance and Spanish Golden Age literature, several projects of a high philological level and digital value have been carried out. This article intends to review some of the most emblematic and committed projects to digital humanities, and to evaluate to what extent they fit with the good practices of digital scholarly edition.

\section{Keywords}

Digital scholarly editions; Spanish Golden Age

En los últimos veinte años, la edición académica ha afrontado el reto de su transformación y adaptación digital con más éxito en algunos casos que en otros. En cuanto que disciplina central en el campo de los estudios humanísticos, la edición ha sido también uno de los pilares de las humanidades digitales y uno de los campos pioneros. ${ }^{1}$ Han florecido cuestiones teóricas en torno a la diferente naturaleza del medio electrónico y al nuevo ecosistema en que se inscribe, todo ello - como no podía ser de otra manera - acompañado de casos prácticos y de publicaciones en línea. En toda esta vorágine, las diferentes tradiciones se han ido adaptando, en la medida que les ha sido posible, a las prácticas digitales procedentes, mayoritariamente, del mundo anglosajón. En el ámbito de la hispanística, las iniciativas digitales relacionadas con la Edad Media y el Siglo de Oro han sido abundantes, especialmente para este último período. ${ }^{2}$

1. Ente las muchas lecturas a propósito de la relación entre humanidades digitales, véase Earhart (2012). La idea es recogida también por Pierazzo: «In the same way that textual scholarship is an interdisciplinary component of the Humanities (one edits texts in literature, history, philosophy, classics and so on), digital textual scholarship represents its counterpart within the Digital Humanities» (2015: 5).

2. Sobre la edición académica digital en español existen ya diversas contribuciones, de las que solo cito: Lucía Mejías (2008) y sus múltiples trabajos; Fradejas (2009-2010); Revenga (2014) y Spence (2014). 
El objetivo del presente artículo es ofrecer una reflexión general sobre el panorama actual de la edición académica digital en las letras renacentistas, al mismo tiempo que se encuadra en una perspectiva global. En primer lugar, se ofrecen algunas cuestiones todavía en curso de discusión y relativas a la naturaleza y a la evaluación de la edición digital; a continuación, se examinan diversos ejemplos en el campo de la edición, y, finalmente, se proponen algunas rutas por las que debería navegar la edición digital académica.

Una de las primeras cuestiones que surgen al afrontar la edición digital es la falta de modelos y prácticas consolidadas. A diferencia de los modelos impresos, no existe todavía una idea clara de cómo debe ser una edición digital, y eso dificulta tanto la concepción, como la ejecución y la implementación de cualquier proyecto editorial (Price 2009; Gabler 2010). No es una coincidencia que en muchos casos se esté hablando todavía de la era del incunable digital (Dahlström 2000), pues efectivamente, aunque son muchas las características que pueden compartir, cada edición digital difiere de las demás y no hay ninguna igual, especialmente en la presentación de los contenidos textuales.

Lo que sí parece claro, a estas alturas, es la necesidad de dejar atrás la concepción instintiva de las ediciones académicas digitales como reproducciones de los libros impresos o como «máquinas de simulación» (McGann 2004: 201). Las ediciones han sufrido una transformación básica y es que ahora son concebidas no solo como un receptáculo textual, acompañado o no de un estudio, sino más bien como una especie de archivo capaz de tener unas dimensiones mucho mayores y de facilitar una investigación textual a gran escala (MLA 2011). Las ediciones han tomado, pues, una nueva forma donde la centralidad es compartida tanto por el texto como por los paratextos. No es solo la ejecución metodológica de la (re) construcción y presentación del texto que es repensada, sino también el papel de todos esos materiales tradicionalmente conectados con la fuente primaria, siempre utilizados y consultados por el editor, que no podían ser incluidos en un formato impreso. Paralelamente, esta transformación bajo forma de «archivo» ensancha los contextos en que las ediciones digitales son utilizadas, no solo en la investigación académica, sino también en marcos de divulgación o actividades pedagógicas. A ello se suma la aparición de otros nuevos modos de concebir cuestiones tradicionales, como el uso de herramientas de colación automática en la reconstrucción de la tradición textual, y la presentación de los resultados bajo forma de un aparato crítico que se aleja del paradigma clásico a pie de página (Apollon y Bélisle 2014).

Quedan, asimismo, cuestiones abiertas y difíciles de resolver sobre la naturaleza de las ediciones académicas digitales; por ejemplo, el hecho de si estas son o no concebidas para una lectura prolongada o, por el contrario, solo como referencia; o si deben ser destinadas a un público genérico o a uno especializado (Tanselle 2006: 4). Pierazzo señala que, en general, los recursos digitales son más bien objetos para ser utilizados que textos para ser leídos (2015: 147-151). A esta oscilación del "para qué» o el "para quién» pueden sumarse otras observaciones como las de Dot Porter, que registraba el escaso uso de las ediciones digitales por parte de los mismos investigadores: 
The results of my survey bear out the continued usefulness, or at least continued use, of print editions: medievalists are using print editions more than they are using digital editions, and the use of digital editions has not grown over the past nine years, as it has, for example, for digital journals. (Porter 2013: 8)

El hecho, pues, de que los investigadores sigan utilizando primariamente las ediciones en papel debería hacernos reflexionar en el uso que queremos dar a nuestros textos y cuáles deberían ser las expectativas y las necesidades digitales de los usuarios. Seguramente esta desconfianza es fruto todavía del poco número de ediciones en línea, de la falta de criterios claros para su ejecución y presentación, y de la proliferación de textos en línea que, desde un punto de vista académico, carecen de los elementos esenciales exigidos por la ecdótica tradicional.

Creo, además, que hay otra cuestión relevante y es justamente la práctica de la crítica textual que, a lo largo de la historia, ha cuajado en las diferentes escuelas, desde Lachmann a la critique génétique o la filologia d'autore. Cada una de ellas ha ofrecido diferentes resultados editoriales: la tradición neolachmaniana apostaba por la reconstrucción de un texto genuino y de ahí la idea de edición crítica, los historiadores han preferido las ediciones diplomáticas y fieles al documento original, la critique génétique ha privilegiado el proceso creativo que da lugar a un texto, etc. Simultáneamente, se han repensado sus principios desde una perspectiva digital. Pero quizás, como propone Pierazzo (2015: 25-28), es hora de redefinir estas prácticas diferentes a la luz de las potencialidades digitales. El paradigma editorial digital está cambiando y algunos procedimientos, tales como el proceso de marcado, borran las líneas entre las diferentes escuelas de crítica textual. Por ejemplo, una perspectiva diplomática puede coexistir perfectamente con una neolachmaniana: podemos ofrecer una edición diplomática de cada testimonio, mientras reconstruimos simultáneamente el texto ideal. La realidad es que la labor editorial en el medio digital puede entrelazarse de una manera mucho más compleja y profunda con conceptos como la intención del autor, el proceso de creación, la tradición y la recepción textual (Robinson 2013: 107). En este sentido, estamos lejos todavía de haber intuido el alcance de la transformación.

Paralelamente a estas redefiniciones metodológicas han aparecido nuevos modelos de edición que son todavía difíciles de categorizar pero que, en algunos casos, responden al uso de los medios sociales y adoptan dinámicas ajenas. Se habla hoy en día, por ejemplo, de «edición social» (Siemens 2012) para referirse a aquellas ediciones que hacen uso de las herramientas propias de los medios sociales y permiten a los usuarios contribuir con nuevo contenido a través de anotaciones, comentarios, traducciones o transcripciones (el caso más conocido es $A$ Social Edition of the Devonshire MS (BL Add 17,492). ${ }^{3}$ La técnica del crowdsourcing, que se beneficia también de las redes sociales, ha sido adoptada para llevar a cabo tareas de transcripción

3. Véase <https://en.wikibooks.org/wiki/The_Devonshire_Manuscript>. 
de grandes archivos textuales y, por lo general, por instituciones culturales que no disponen del personal suficiente para implementar el proyecto (como la edición Transcribe Bentham). ${ }^{4}$ Aunque no necesariamente es siempre así, como en el caso del Pedrolo Digital, que se inscribe en el marco de una tesis de doctorado. ${ }^{5}$ Han surgido también iniciativas interesantísimas como las reconstrucciones textuales filogenéticas que, como si emularan los patrones de la biología humana, establecen una red de conexiones entre las diferentes variedades de un texto. ${ }^{6}$ Además, y en la línea de la crítica genética, han aparecido ediciones basadas en dosieres genéticos especialmente utilizados en la tradición francesa, ${ }^{7}$ aunque también en algunos manuscritos modernos en lengua castellana, como el Manuscrito digital de Juan Goytisolo. ${ }^{8}$ En fin, existen las ediciones más conocidas que Pierazzo ha llamado "paradigmáticas». Este tipo se basa en un sistema que separa el código fuente de su presentación final (el llamado método source-output), donde el editor es también el codificador del texto y donde encontramos tanto ediciones diplomáticas como ediciones críticas digitales. ${ }^{9}$ Este es el modelo más utilizado en tierras hispanas.

Otra variable que debería tomarse en consideración es el uso de la tecnología en su dimensión heurística y hermenéutica, es decir, en la capacidad de hallazgo y de un nuevo análisis, especialmente en el establecimiento del texto a partir de la práctica digital (Gabler 2010). Pierazzo plantea una dualidad basada justamente en el valor hermenéutico del flujo de trabajo digital (2015: 109-117). Por un lado, existe un grupo mayoritario de investigadores que ven en el ordenador solo una herramienta útil para facilitar ciertas tareas de automatización, es decir, se adopta el ordenador pero sin cambiar substancialmente el flujo de trabajo. Hoy en día, a la hora de llevar a cabo una edición, todo el mundo utiliza reproducciones facsimilares, un procesador de texto, y manda una copia digital al editor, según Pierazzo, es lo que Shillingsburg (1996) llamó «computer assisted scholarly editing». Por el otro, el flujo de trabajo completamente digital apuesta por el valor hermenéutico y adopta procesos y metodologías computaciones para producir ediciones digitales interactivas. Imaginemos, por ejemplo, el uso de programas de colación, técnicas de lematización, procesos de reconocimiento de caracteres, el uso de ficheros XML para salidas en formatos diversos, entre un largo etcétera. Esta última concepción es la que Gregory Crane, entre otros, ha llamado ePhilology, y por la que deberíamos apostar, aunque sea el camino más desafiante (Crane, Bamman y Jones 2007).

\footnotetext{
4. Véase <http://blogs.ucl.ac.uk/transcribe-bentham/>.

5. Véase $<$ http://pedrolodigital.pfdorado.com/wp/>.

6. Véase por ejemplo el proyecto Stemmaweb en <https://stemmaweb.net/>.

7. Por ejemplo, Les manuscrits de Madame Bovary en <http://www.bovary.fr/>, o Les manuscrits de Stendhal en <http://manuscrits-de-stendhal.org/>.

8. Véase <http://goytisolo.unibe.ch/>

9. Para una explicación detallada sobre esta tipología véase Pierazzo (2015: 25-29). También en el artículo (Allés Torrent 2015) se explica este tipo de flujo de trabajo.
} 
En lo que atañe a la proliferación de ediciones digitales a nivel internacional, recordaré que actualmente existen dos grandes catálogos: uno es el Catalogue of Digital Scholarly Editions de Patrick Sahle (Universität zu Köln) y el Catalogue of Digital Editions de Greta Franzini (University of Göttingen). ${ }^{10}$ El primero contiene solo cinco títulos en lengua española. ${ }^{11}$ En el de Franzini, que adopta una definición más amplia de edición digital, se observa un panorama internacional multilingüe donde, de un total de 187, hay unas 60 en lengua inglesa, seguido por 44 en lengua latina, 12 en francés, 10 en italiano y solo 7 en español (Franzini, Terras y Mahony 2016: 176). ${ }^{12}$ La presencia pues de ediciones digitales en lengua española en estos catálogos es realmente escasa; el punto positivo es que la mayoría de ellas pertenecen justamente a ediciones de textos medievales y del Renacimiento.

\section{En busca del equilibrio con la tradición}

$\mathrm{Al}$ explorar las causas que han contribuido a esta escasez de ediciones digitales podría culparse, en primer lugar, a la falta de una preparación técnica de los estudiosos. Aunque este panorama está cambiando muy rápidamente, es seguramente la ignorancia del flujo del trabajo digital lo que ha dificultado la introducción del componente hermenéutico en nuestros estudios. A ello se le suma el problema de la evaluación del trabajo digital, en muchas ocasiones todavía considerado como una actividad técnica y no siempre reconocida como producción académica. Además, la dependencia de financiaciones que duran solo pocos años juega en contra de la misma naturaleza digital que es siempre cambiante y necesita de actualizaciones constantes. Por último, y no menos importante, existe la deuda y el peso de la tradición; todavía falta una propuesta orgánica que reúna tradición e innovación, digamos, cómo casar la práctica de Menéndez Pidal con una hermenéutica digital.

La relación con la tradición no es un problema nuevo. Desde hace largo tiempo se ha venido discutiendo la necesidad de renovar, en general, la disciplina filológica. Ya en los años 90 Roberto Busa, considerado por muchos el precursor de las humanidades digitales por su relación con IBM y la informatización del Index Thomisticum, habló sobre la pertinencia de una «nueva filología»:

10. Los catálogos pueden encontrarse en las siguientes direcciones: <http://www.digitale-edition. de/> y <https://dig-ed-cat.acdh.oeaw.ac.at/>.

11. Estos títulos son: The Alcalá Account Book, La Entretenida by Miguel de Cervantes, Manuscrito digital de Juan Goytisolo, Códice Mendoza, Electronic Variorum Edition of the Quixote, Edición critica y archivo digital de La dama boba, Torquemada en la hoguera de Benito Pérez Galdós.

12. En la tabla disponible en GitHub aparecen tres títulos más, un total de diez: Becerro Galicano de San Martín de la Cogolla (aunque escrito en latín), La Entretenida, Electronic Variorum Edition of the Quixote, Quijote Interactivo, Orlando Furioso Hypertext, Cantar de Mio Cid, An Electronic Corpus of 15th Century Castillian Cancionero Manuscripts, The Alacala Account Book Project, The Pérez Galdós Editions Project, Cancionero digital de Gómez Manrique. 
I am speaking of a "new" philology, in an evolutionary but not revolutionary sense. I may describe it either as the one which points to the micro-analysis of our macrocognitive processes, or -and it is the same- the one which points to formalize for computer use the global meaning of textual sets. (Busa 1992: 131)

Según Busa, pues, corresponde a los humanistas el discernir en qué consiste nuestro proceso cognitivo y de qué manera lo podemos traducir y formalizar en términos informáticos. Y es ahí donde reside la cuestión y es ese el objetivo que deberíamos perseguir, no solo al referirnos al texto, como datos textuales, sino a todos los otros artefactos culturales, ya sea cultura visual, literatura, o media studies.

La idea de la ePhilology, antes mencionada y propuesta por Crane, es particularmente indicada para designar un cambio de rumbo en la disciplina que encaje, por un lado, con una definición generalista de una disciplina que pretenda continuar dando sentido a los textos, y por el otro, con una práctica y evolución digital. En este sentido, pienso que deberían tenerse en cuenta al menos tres principios: por un lado, el mantener la centralidad del texto; por el otro, la interiorización de que los textos son datos informáticos, es decir, ser consciente de la necesidad de trabajar con bits y bytes para alimentar el ordenador y entender su dinámica; y en fin, la aplicación de un pensamiento algorítmico a nuestra práctica. No obtendremos nunca ningún proceso automático o programa aplicable a nuestro campo, si antes no lo hemos pensado nosotros en nuestra calidad de humanistas.

La verdad es que estamos todavía construyendo el equilibrio entre lo que ha sido nuestra formación académica y las nuevas tecnologías. A lo largo de este proceso encontramos tanto investigadores escépticos como optimistas incondicionales, y estamos aún a las puertas de lo que hemos llamado la hermenéutica digital. Los cambios llegarán, pero es importante que, como decía Busa, se conciban más bien como una evolución que no como una revolución.

Dicho esto, también debo subrayar que no ha habido una falta de interés en el medio digital en los estudios de hispanística. Alberto Blecua, por ejemplo, en un artículo de 1991, destacaba con especial interés una edición del Libro de Alejandro publicada en 1987 por el Prof. Marcos Marín; la principal novedad residía justamente en la ejecución digital y en el hecho que era elaborada con la ayuda de un ordenador (Blecua 1991: 78). La edición no se concebía como una «edición crítica» en el sentido tradicional, sino que —en palabras de Marcos Marín - la metodología empleada permitía preservar «el rico polimorfismo de las formas gráficas en un manuscrito del siglo XIV» (1987: 479). El objetivo principal de esa edición era ofrecer una «nueva metodología» respetuosa con los valores lingüísticos y los conocimientos adquiridos, pero no restringida por los diseños tradicionales de la presentación del texto. Otros estudios, hoy considerados pioneros, florecieron en los siguientes años, como los trabajos del mismo Francisco Marcos Marín (1994) y el de Estelle Irizarry (1997).

Lo que es incuestionable, al menos para mí, es la relevancia de los modelos críticos heredados. Estoy de acuerdo con Thomas Tanselle cuando dice que los 
objetivos de la edición académica no cambiarán debido a la tecnología digital, sino que estos deberían permanecen inalterados, es decir, el hecho debe aspirar a la preservación, el acceso, la diseminación, el análisis y la interpretación, de la misma manera que tampoco la formalización ni el método crítico básico se alteran. Pero Tanselle también observaba - y no sin recibir algunas críticas - que cuando el entusiasmo digital llevaba a la idea de que los ordenadores alterarían la ontología de los textos y que posibilitarían nuevos tipos de lectura y de análisis se había llegado demasiado lejos. ${ }^{13}$ Sea como sea, como sugieren Buzzetti y McGann (2004: 54), aunque nuestros objetivos tradicionales permanezcan, las nuevas tecnologías nos están obligando a revisitar y repensar algunos de los problemas más básicos de la textualidad y la teoría del texto, pues la edición impresa y la digital difieren dramáticamente tanto en la concepción como en la ejecución.

En fin, también David Greetham afirma que hay que reconocer el valor de ciertos principios básicos heredados (como el papel del autor, la función de la audiencia, la variación en los textos), que deben continuar guiando tanto los procedimientos como los juicios sobre la práctica de la crítica textual, pero que debemos reconocer a su vez que los nuevos materiales, los métodos de almacenamiento y transmisión y los nuevos principios críticos tendrán que formar parte de las competencias del investigador en un futuro inmediato (Greetham 2013: 39-40).

\section{La cuestión (ir)resuelta de la evaluación}

Se han dedicado muchos esfuerzos a la evaluación de la investigación digital en general, dando un conjunto de buenas prácticas y tratando problemas como la autoría y la colaboración (Presner 2012; MLA 2012).

La crítica textual tradicional e impresa siempre ha ofrecido un conjunto de reglas estrictas, tanto de método como, sobre todo, de presentación. Este no es el caso de la crítica textual digital, aunque sí se han formulado algunas directrices generales. Desde 2011, la Modern Language Association ha estado actualizando sus Guidelines for Editors of Scholarly Editions y ha incluido un conjunto de puntos relacionados con temas digitales. Todos los criterios válidos para las ediciones académicas tradicionales son aplicables para su contraparte digital, con el objetivo de presentar un texto fiable establecido a partir de la exactitud, la adecuación, la consistencia y la explicitud. En sus directrices generales, la MLA tiene en cuenta cuatro cuestiones principales: el uso de estándares, la codificación del texto, el uso y manipulación de imágenes y la documentación digital, de la siguiente manera:

13. Estas son sus palabras «hyperbolic writing and speaking about the computer age, as if the computer age were basically discontinuous with what went before, $[\ldots]$ when the excitement leads to the idea that the computer alters the ontology of texts and makes possible new kinds of reading and analysis, it has gone too far.» (Tanselle 2006: 2) 


\begin{tabular}{|c|c|c|c|}
\hline Uso de estándares & Imágenes & Codificación & Documentación \\
\hline $\begin{array}{l}\text { - Gramáticas estándares } \\
\text { ISO: codificación de } \\
\text { caracteres (Unicode), } \\
\text { instrucciones de } \\
\text { transformación (ej. } \\
\text { XSLT), imágenes } \\
\text { digitales (JPEG, PNG), } \\
\text { audio y video (MPEG, } \\
\text { MP3), etc. } \\
\text { - Metadatos técnicos, } \\
\text { descriptivos y } \\
\text { administrativos para } \\
\text { todos los elementos }\end{array}$ & $\begin{array}{l}\text { - Elementos multimedia } \\
\text { (imagen, sonido, video) } \\
\text { de alta resolución, } \\
\text { almacenados en un } \\
\text { tamańo razonable. } \\
\text { - Permitir la } \\
\text { manipulación de las } \\
\text { imágenes (agrandar y } \\
\text { minimizar). }\end{array}$ & $\begin{array}{l}\text { - Ficheros codificados en } \\
\text { un formato abierto y no } \\
\text { propietario y gramáticas } \\
\text { estándares ISO (ej. TEI- } \\
\text { XML, y no Microsoft } \\
\text { Word o PDF). } \\
\text { - Adopción de la } \\
\text { codificación a partir de } \\
\text { las líneas directrices de } \\
\text { una comunidad. }\end{array}$ & $\begin{array}{l}\text { - Ayuda al usuario (para } \\
\text { usar la interfaz) } \\
\text { - Explicación y puesta a } \\
\text { disposición de: } \\
\text { - la codificación (ficheros } \\
\text { de texto), } \\
\text { - la DTD o esquema, o } \\
\text { ODD, } \\
\text { - la hojas de } \\
\text { transformación, } \\
\text { - esquema de la base de } \\
\text { datos. } \\
\text { - Indicación clara de la } \\
\text { licencia, y permisos } \\
\text { en la reutilización del } \\
\text { material. } \\
\text { Disponibilidad del } \\
\text { código fuente del } \\
\text { programa desarrollado } \\
\text { para la edición. }\end{array}$ \\
\hline
\end{tabular}

Otra propuesta formal es ofrecida por Patrick Sahle y el equipo del Institut für Dokumentologie und Editorik (IDE) de Colonia. Para ellos, una edición académica, tanto impresa como digital, es la representación crítica de documentos históricos que debe cumplir con los paradigmas académicos tradicionales (Sahle 2008-2016). Establecen, en primer lugar, una clara distinción entre una edición digitalizada y una edición digital. La primera se refiere a una mera publicación en formato digital que incluye ediciones impresas digitalizadas (facsímiles o formatos tipo PDF) o proyectos de digitalización de bibliotecas, incluso cuando tienen un conjunto completo y correcto de metadatos. Asimismo, proponen como definición mínima que una edición digital no se puede imprimir sin una pérdida de información y/o de funcionalidad (es decir, si lo que obtenemos al imprimir es el mismo resultado que las opciones que tenemos en pantalla, no es una edición académica digital). En definitiva, defienden que la edición digital está guiada por un paradigma diferente al espacio bidimensional de la "página» impresa y a los medios tipográficos de representación de la información.

El mismo equipo, en su revista dedicada a reseñas de ediciones digitales (A Review Journal for Digital Editions and Resources), ofrece una sección para los revisores llamada Criterios para la reseña de ediciones digitales académicas, ahora disponible en español y que aspira a establecer una lista de condiciones y recomendaciones para la evaluación de las ediciones en línea. ${ }^{14}$ Esta serie de criterios profundiza en el análisis y constituye la guía utilizada por sus revisores, al mismo tiempo que da algunas pistas sobre este «paradigma diferente» de la edición digital.

14. Disponible en: <http://www.i-d-e.de/publikationen/weitereschriften/criterios-version-1-1/> . 


\section{Edición digital y Siglo de Oro}

En los estudios ibéricos tanto medievales como del Renacimiento, la tendencia general ha sido la creación de corpus y bases de datos o catálogos, y proyectos generales centrados en estudios filológicos (Spence 2014).

Los corpora han proliferado especialmente en el ámbito de los estudios medievales; seguramente debido a la relevancia del estudio de la lengua en esta época. La mayoría de las veces dichos proyectos no son simplemente corpus stricto sensu, como sucedía con ADMYTE o el propio CORDE, sino que tienen otras funcionalidades, incluyendo la presentación de los textos, como es el caso del Electronic Corpus of the $15^{\text {th }}$ Castilian Cancionero Manuscript, Reperterio Métrico Digital de la Poesía Medieval Castellana, el proyecto Biblia Medieval, CHARTA y el Corpus des Troubadours.

Los catálogos y bases de datos, por otro lado, comenzaron a construirse desde los años noventa, siguiendo el camino abierto por el proyecto Philobiblion y Charles Faulhaber. Hoy en día estos constituyen la gran mayoría y podemos - por mencionar solo unos pocos- consultar bases de datos sobre la literatura medieval ibérica (como las debidas al grupo Clarisel), sobre la literatura medieval catalana (proyecto NARPAN) o sobre las traducciones, como el pionero Proyecto Boscán, o últimamente el Catálogo Hipertextual de Traducciones Anónimas al Castellano.

Desde mi punto de vista, el principal problema con los corpora es el hecho de que no ofrecen todavía acceso al texto completo. La mayoría de ellos no tienen una licencia de libre acceso ni código abierto, ni poseen una API donde ejecutar solicitudes, ni ofrecen archivos descargables en texto plano o en XML. Por su lado, las bases de datos reflejan en muchos casos el método tradicional de recolección de información en fichas, donde cada entrada carece de interactividad, el uso de datos enlazados es raro y el software de base de datos varía, pasando de los propietarios (Filemaker) a otros de código abierto (MySQL).

Varias bibliotecas digitales también han visto la luz, la mayoría de ellas esta vez centradas en el período de la Edad de Oro, como la Biblioteca Áurea Digital (BIADIG), la Bibliografia de Escritoras Españolas (BIESES), la Biblioteca Digital del Siglo de Oro (BIDISO), el Portal Andrés de Poza, o la Biblioteca Digital de Diálogo Hispánico (DIALOGYCA).

Hay proyectos que conjugan catálogo y biblioteca digital como ARTELope Biblioteca Digital, y otros que se han ramificado en múltiples iniciativas digitales como el ya mencionado BIDISO (López y Saavedra 2014). Ha habido, incluso, proyectos que se han unido constituyendo una red de iniciativas afines, como la Red Aracne (Baranda y Rodríguez 2014), o TC/12 Patrimonio teatral clásico español, que agrupa diversos equipos de investigación comprometidos con la digitalización y la edición del patrimonio teatral, como es el caso del grupo ProLope.

En líneas generales, este tipo de textos, como los ofrecidos por la Biblioteca Virtual Miguel de Cervantes, limitan su presentación a simples páginas HTML, proporcionando mayoritariamente el texto de una edición impresa, una transcripción fiel o incluso un PDF. Otros como la Biblioteca ArteLope (Muñoz 
Pons 2013) ofrecen posibilidades más sofisticadas como la visualización de los elementos estructurales de las obras teatrales, desde los datos de la edición, el responsable de la marcación digital, los elementos paratextuales, como dedicatorias, la estructura en actos, las acotaciones, apartes, versos partidos o incluso la métrica. Asimismo, la Biblioteca Digital de la Literatura de l'Edat Moderna del proyecto NISE ha inaugurado una serie muy prometedora de ediciones de autores catalanes, como la de Francesc Fontanella, donde el texto crítico convive con diversas secciones, como variantes textuales, comentarios a dichas variantes, notas editoriales, además de un extenso apartado de metadatos. ${ }^{15}$

Como se ha dicho, una edición digital debería ofrecer los resultados de un estudio interpretativo de un texto y aportar un conocimiento nuevo sobre el mismo (Franzini, Terras y Mahony 2016: 162). En este sentido, las ediciones facsímiles son una mera réplica del original y, aunque utilísimas, no pueden ser consideradas una edición digital propiamente dicha. Lo mismo sucede con los resultados en PDF, que continúa siendo un formato destinado básicamente para ser impreso, hecho que, según Patrick Sahle, va en contra de la misma naturaleza digital.

En el caso de la producción del Siglo de Oro, grandes proyectos financiados sobre Lope de Vega y la producción teatral han llevado a la construcción de algunas ediciones digitales académicas (Spence 2013). Me gustaría destacar el valor especial de dos obras: la edición digital de La Entretenida de Miguel de Cervantes, dirigida por John O'Neill (King's College) y La Dama Boba de Lope de Vega, dirigida por Marco Presotto (Università di Bologna). El primero ofrece cuatro versiones del texto español que permiten diferentes visualizaciones de la puntuación y la ortografía (facsímil, transcripción ortográfica moderna, edición por O’Neill y otra no puntuada), junto con una traducción al inglés llevada a cabo por el mismo editor. Yo diría que es un excelente ejemplo de edición académica digital, pero se echan de menos dos cosas: la disponibilidad de los archivos codificados en XML-TEI, y la imposibilidad de ver simultáneamente el facsímil y una versión, o una versión y la traducción, o incluso varias versiones simultáneamente. La Dama Boba es también un gran ejemplo que ofrece una nueva edición digital crítica, centrándose principalmente en la historia del texto juntamente con un amplio estudio. Además, la edición tiene una buena documentación técnica y la mayoría de los códigos fuente descargables. ${ }^{16}$

Conviene también recordar los proyectos relacionados con el Quijote: Electronic Variorum Edition of the Quixote (EVE-DQ), auspiciado por Eduardo Urbina, y el Quijote interactivo, dirigido por José Manuel Lucía Megías y albergado por la Biblioteca Nacional de España. Este último responde claramente a la no-

15. Disponible en: $<$ http://www.nise.cat/BibliotecaDigital/Autors/Autor/3/Fontanella_Francesc.aspx $>$. 16. Ambas obras han sido objeto de reseñas en RIDE. Véanse Allés Torrent (2016) y Rojas Castro (2017). En el caso de La dama boba puede descargarse el código XML-TEI del texto crítico en: $<$ http://damaboba.unibo.it/xml/edicion_critica.xml>. 
ción de archivo digital, pues acumula una gran variedad y riqueza de elementos paratextuales, tales como imágenes y mapas, así como materiales destinados a un uso contextual mayor, más allá del ámbito académico.

Otras iniciativas se han adentrado en el complejo territorio de la edición de traducciones y las versiones múltiples. Hace ya algunos años el Hipertexto del Orlando Furioso, dirigido por María de las Nieves Muñiz Muñiz, ofrecía el texto italiano de Ariosto y el texto español en traducción de Jerónimo de Urrea con una serie de materiales facsimilares; el Portal Andrés de Poza, liderado por Carmen Isasi, por su lado, está dedicado a la edición de textos literarios europeos con versiones múltiples del ámbito vasco-románico entre los siglos XVI y XVII.

En fin, es obligatorio mencionar otras iniciativas como el Sitio de Guaman Poma, albergado en la Det Kongelige Bibliotek en Copenhage, que ofrece la reproducción facsimilar del códice acompañada de una transcripción y notas de comentario; y especialmente el Códice Mendoza, una original propuesta llevada a cabo por el Instituto Nacional de Antropología e Historia, que ha transformado un manuscrito mexicano, confeccionado en 1542, en una edición digital explotando el aspecto visual del códice original.

Durante los últimos congresos de humanidades digitales en español ha habido múltiples comunicaciones proponiendo proyectos editoriales digitales. De nuevo, una gran mayoría de los trabajos tocaban obras del Siglo de Oro de autores como Pedro Calderón de la Barca, Tirso de Molina, o Luis de Góngora: como la Edition digitale et étude de la polémique autour de Góngora, o la edición de las Soledades. ${ }^{17}$

Ante esta serie de iniciativas, me gustaría señalar dos cuestiones: la primera es que muchos de estos proyectos se presentaron como empresas individuales, es decir, donde los editores, la mayoría de ellos doctorandos, se enfrentan a las tareas de modelado y codificación: los editores ya se han convertido en los codificadores. La segunda es que todos ellos planeaban utilizar lenguajes estándares, siendo el lenguaje más utilizado el XML basado en las directrices de la Text Encoding Initiative y el uso del lenguaje XSLT para realizar las transformaciones de salida. En este panorama parece vislumbrarse la tendencia de las generaciones futuras, capaces de realizar trabajos textuales y digitales, integrando la dimensión hermenéutica de la tecnología en su práctica cotidiana.

\section{Limitaciones y retos}

El panorama dibujado es sin duda prometedor, pero queda todavía mucho recorrido por avanzar en la edición digital de los textos hispánicos. En este sentido, creo que deberíamos prestar atención a todas y cada una de las etapas que entran

17. Las descripciones de estos dos proyectos en ciernes sobre Góngora pueden encontrarse en: <http://obvil.paris-sorbonne.fr/corpus/gongora/>, <http://www.antoniorojascastro.com/proyectos-de-humanidades-digitales/edicion-digital-soledades $/>$. 
en juego a la hora de ejecutar un proyecto editorial digital. Me refiero a las fases de modelado de datos, los procesos de transcripción, marcado y edición, la publicación final, y la infraestructura digital.

En primer lugar, el modelado de datos debería liberarse de la condición pedisecua de la página impresa y ser el punto de partida donde residiera la capacidad para redefinir las características textuales, más allá de su estructura — pongamos por caso- en capítulos y párrafos, y se concibieran las posibilidades digitales del modelo textual (Earhart 2012; Crane, Bamman, Jones 2007). Esta premisa se conecta con la idea de que toda edición digital debería concebirse como un objeto dinámico: allí donde la edición papel «congela» o establece un texto para ser difundido - fruto obviamente de una minuciosa reflexión y una serie de pautas-, la edición digital asienta las bases para que ese texto empiece una andadura digital a través, por ejemplo, de la colaboración, o de la reutilización de su código.

Aparte del modelo de datos, la edición digital de textos hispánicos debería apostar definitivamente por un marcado semántico. En cierta medida, actualmente los marcados digitales dependen todavía en muchos casos de la página impresa, y es lógico: la literatura nace estrechamente ligada a una cultura material concreta. Pero, como hemos visto, una edición digital debe proporcionar nuevo conocimiento, más allá de replicar lo que ya había en la página original. Un marcado semántico abre, además, la puertas a una serie de técnicas que ayudan a la explotación del material textual tales como reconocimiento de entidades nombradas, detección de alusiones, anotaciones lingüísticas de diferente naturaleza, como la lematización, o las alineaciones de traducciones.

Como comunidad deberíamos practicar no solo el acceso abierto - especialmente cuando se trata de resultados obtenidos con fondos públicos- sino también el código abierto. Proyectos como el Becerro Galicano, La dama boba o el Análisis distante del soneto castellano de los Siglos de $\mathrm{Oro}^{18}$ ayudan a compartir, difundir y consolidar prácticas en materia de marcado; y no solo eso, abren un sinfín de posibilidades para que otros investigadores trabajen y exploren los datos desde un punto de vista digital.

En tercer lugar, deberíamos prestar especial atención al resultado de las publicaciones. Los usuarios somos cada vez más exigentes en lo que se refiere a ergonomía, accesibilidad y diseño. Eso nos lleva al problema de las interfaces, difíciles de concebir, diseñar y construir. A diferencia de la codificación donde el editor es o puede ser el codificador y donde los métodos filológicos son fácilmente asequibles de aprender en poco tiempo, pues permanecen dentro de los límites del pensamiento editorial tradicional, el diseño web es un área completamente diferente. $\mathrm{Al}$ no haber un patrón estándar de modelo de edición, conviene reflexionar continuamente en cuál es la interfaz más amigable, más interactiva, flexible e intuitiva.

18. Estos proyectos pueden encontrarse en: <http://www.ehu.eus/galicano/?l=en>, y en $<$ https:// adsoua.wordpress.com/>. 
Muchas variables entran en juego, y para ello es imprescindible pensar en lo que los usuarios querrán hacer con nuestros materiales, no solo con los textos, sino también con el paratexto y todas las fuentes suplementarias. La experiencia del usuario puede ir mucho más allá de la simple lectura o consulta de la obra, que, por lo demás, se ha demostrado no siempre cómoda (Pierazzo 2015: 158168). El mundo digital, pues, nos obliga a pensar de una manera más abstracta y a reflexionar tanto en el resultado que el usuario visualizará como en las formas en que podrá aprovechar nuestro recurso.

La adopción de ciertos principios del diseño web podría brindar perspectivas interesantes a la hora de concebir nuevas interfaces; como indica Pierazzo (2015: 158-168), el esqueumorfismo, en términos de diseño web, consiste en el intento de imitar digitalmente objetos analógicos y de la vida real. El ejemplo típico es la "papelera de reciclaje» o el concepto de «escritorio» de nuestras computadoras. Quizás sería conveniente plantearnos un diseño diferente de lo que venimos haciendo — que no consistiría en la reproducción de la página escrita sino en la recreación de una "estación de trabajo» ideal para el editor/lector, por ejemplo, el hecho de tener todas las copias del texto a su alcance-. O, ¿por qué no pensar en términos de interacción $3 \mathrm{D}$ con los textos?

Se ha señalado también que la falta de un modelo estándar y el hecho de que cada edición presenta una interfaz de usuario diferente es desorientador y en última instancia desagradable para los investigadores que están acostumbrados al uso de obras impresas (Rosselli Del Turco 2011). En un libro impreso sabemos siempre dónde está el índice (al principio o al final), o dónde están las notas o el aparato crítico; pero esto no sucede en la edición digital, donde siempre tenemos que invertir algún tiempo para darnos cuenta de dónde está cada sección.

En fin, debemos también tomar en consideración todo lo relativo a las infraestructuras que albergan los proyectos digitales, ya sea de edición digital o de otra naturaleza. En este sentido, creo que toda infraestructura debería ser minimalista y creada con tecnologías estándares y libres: texto plano, XML, HTML, programario libre, etc. de manera que todo el mundo pudiera tener acceso sin la necesidad de ningún programa sofisticado ni propietario. Eso incluye, en algunos casos, recapacitar sobre el uso de ciertos plugins y entornos, por ejemplo, que dificultan la consulta de algunas ediciones especialmente en ciertos navegadores. Como la MLA (2011) ha sugerido, las tecnologías empleadas son tan importantes como el método que implementamos y ninguna decisión técnica está libre de implicaciones metodológicas y viceversa.

La mayoría de los proyectos digitales son works in progress que requieren una infraestructura escalable, es decir, que permita el crecimiento del proyecto. Plataformas como GitHub, por ejemplo, están siendo cada vez más utilizadas porque pueden funcionar a la vez como repositorio del código fuente y como método de publicación.

El todo creo que pasa también por una apuesta contundente por la reutilización y la reproducibilidad en un sentido de ida y de vuelta: antes de reinventar 
la rueda, es mejor asegurarse si hay ya paquetes de código que nos puedan ser útiles, por ejemplo, el uso de frameworks para los sitios web. Y viceversa, que lo que nosotros hagamos pueda ser reutilizado por otros con la debida licencia y mención.

Creo también - y este parece ser un aspecto no contemplado entre las ediciones de la literatura hispánica medieval y del Siglo de Oro- que merecería la pena invertir nuestra labor intelectual en el aspecto social del texto y en la colaboración. La naturaleza intrínseca del medio digital hace necesaria una colaboración tanto en las fases de ejecución como en las de difusión.

En fin, ańadiré otros dos aspectos que me parecen esenciales: por un lado, la apuesta por la preservación a largo término: una vez el proyecto ha «concluido" es necesario seguir destinando esfuerzos para el mantenimiento del sitio y los datos, que idealmente debería ser realizado por los mismos humanistas responsables. Por el otro, insistir en el código abierto: si la investigación debe ser transparente y respaldarse en resultados previos, cualquier trabajo digital también debería serlo porque la labor digital forma parte de la investigación y cada vez más es sometida a evaluación.

Retomando pues el pasaje quijotesco aludido en el título y pronunciado por Sancho, deberíamos tener en cuenta que «entre los estremos de cobarde y de temerario está el medio de la valentía» y plantearnos en qué medida los trabajos a realizar pueden inscribirse en la serie de prácticas expuestas por desafiantes que parezcan. Tanto la literatura medieval como la del Siglo de Oro ofrecen un terreno sumamente fértil en el que poder practicar y aplicar nuevas formas de edición (crítica) digital, modelos más originales de publicación, medios más amplios de difusión y flujos de trabajo centrados en la colaboración. Algunos de los proyectos mencionados y aquellos en curso parecen augurar, en definitiva, que los tiempos de acometer la edición académica digital ya llegaron. 


\section{Bibliografía}

Allés Torrent, Susanna, «Edición digital y algunas tecnologías aliadas», Ínsula, 812 (2015), 18-21.

-, "Cervantes and the Golden Age Theatre: First Attempts Towards a Digital Scholarly Editorial Model», A Review Journal for Digital Editions and Resources, 4 (2016), (25-09-2017), <http://ride.i-d-e.de/issues/issue-4/entretenida/>. Apollon, Daniel y Claire BÉlisle, «The Digital Fate of the Critical Apparatus», Digital Critical Editions, eds. Daniel Apollon, Claire Bélisle, Philippe Régnier, Champaign (IL), University of Illinois Press, 2014, 81-113.

Baranda, Consolación y Eduardo Rodríguez, «Red ARACNE: retos y objetivos de un proyecto de coordinación en letras hispánicas digitales», Janus. Estudios sobre el Siglo de Oro, Anexo 1 (2014), 101-110.

Blecua, Alberto, "Los textos medievales castellanos y sus ediciones», Romance Philology, 45/1 (1991), 73-88.

Busa, Roberto, «Half a Century of Literary Computing: Towards a 'New' Philology", Historical Social Research, 17/2 (1992), 124-133.

Buzzetti, Dino y Jerome McGann, "Critical Editing in a Digital Horizon», Electronic Textual Editing, eds. Lou Burnard, Katherine O'Brien O'Keeffe, John Unsworth, New York, The Modern Language Association of America, 2006, 53-73.

Crane, Gregory, David Bamman y Alison Jones, «ePhilology: When the Books Talk to Their Readers", A Companion to Digital Literary Studies, eds. Ray Siemens, Susan Schreibman, Oxford, Blackwell Publishing, 2007, 29-64.

DAHLSTRÖM, Mats, «Digital Incunables: Versionality and Versability in Digital Scholarly Editions», Electronic Publishing in the Third Millennium, eds. Peter Linde, John Smith, Elena Emelianova, Washington, ICCC, 2000, 224-234.

Earhart, Amy E., «The Digital Edition and the Digital Humanities», Textual Cultures, 7/1 (Primavera 2012), 18-28.

Fradejas Rueda, José Manuel, «La codificación XML/TEI de textos medievales», Memorabilia, 12 (2009-2010), 219-247.

Franzini, Greta, Melissa Terras y Simon Mahony, «A Catalogue of Digital Editions", Digital Scholarly Editing: Theories and Practices, eds. Matthew James Driscoll, Elena Pierazzo, OpenBook Publishers, 2016. $<$ https://doi.org/10.11647/OBP.0095>

Gabler, Hans Walter, «Theorizing the Digital Scholarly Edition», Literature Compass, 7/2 (2010), 43-56.

Greetham, Davir, "A History of Textual Scholarship», The Cambridge Companion to Textual Scholarship, eds. Neil Fraistat, Julia Flanders, Cambridge University Press, 2013, 16-41.

IrIZARRY, Estelle, Informática y literatura: análisis de textos hispánicos, Barcelona, Proyecto A Ediciones, 1997. 
López Poza, Sagrario y Ángeles SaAvedra Places, «Recursos digitales ofrecidos por el SILEAE para el estudio del Siglo de Oro. Creación, gestión y evolución de BIDISO», Janus. Estudios sobre el Siglo de Oro, Anexo 1 (2014), 285-304.

Lucía Megías, José Manuel, «Las relaciones entre la bibliografía textual y la informática humanística: El incunable del hipertexto», Tipofilologia. Rivista Internazionale di Studi Filologici e Linguistici sui testi a stampa, 1 (2008), 119-138.

Marcos Marín, Francisco, Informática y Humanidades, Madrid, Gredos, 1994. McGann, Jerome, «Marking Texts of Many Dimensions», Companion to Digital Humanities, eds. Susan Schreibman, Ray Siemens, John Unsworth, Oxford, Blackwell Publishing, 2004, 198-217.

Modern Language Association, Guidelines for Editors of Scholarly Editions, 29-06-2011, <http://mla.org/cse_guidelines>.

—, Guidelines for Evaluating Work in Digital Humanities and Digital Media, 2012.

-, Statement on the Scholarly Edition in the Digital Age, MLA Commitee on Scholarly Editions, Mayo 2016.

Muñoz Pons, Carlos, «El proceso de edición digital en Artelope y CTCE», Teatro de palabras. Revista sobre teatro áureo, 7 (2013), 483-496.

Pierazzo, Elena, Digital Scholarly Editing. Theories, Models and Methods, Farnham, Surrey; Burlington, VT: Ashgate, 2015.

Porter, Dot, «Medievalists and the Scholarly Digital Edition», Scholarly Editing, 34 (2013), 1-26.

Presner, Todd, "How to Evaluate Digital Scholarship», Journal of Digital Humanities, 1/4 (2012), <http://journalofdigitalhumanities.org/1-4/ how-to-evaluate-digital-scholarship-by-todd-presner/>.

Price, Keneth, «Edition, Project, Database, Archive, Thematic Research Collection: What's in a Name?», Digital Humanities Quarterly, 3/3 (2009), <http://www.digitalhumanities.org/dhq/vol/3/3/000053/000053.html>.

Revenga, Nadia, «La edición crítica digital de textos teatrales. La Estrella de Sevilla como ejemplo", Anuario de Lope de Vega. Texto, literatura, cultura, XX (2014), 99-121.

Rojas Castro, Antonio, «Lope de Vega's La Dama Boba. Critical edition and digital archive», A Review Journal for Digital Editions and Resources, 5 (2017). <https://doi.org/10.6092/UNIBO/LADAMABOBA>

Robinson, Peter, «Towards a Theory of Digital Editions», Variants, 10 (2013), 105-132.

Rosselli Del Turco, Roberto, «After the Editing is Done: Designing a Graphic User Interface for Digital Editions», Digital Medievalist, 7 (2011). $<$ http://doi.org/10.16995/dm.30>

Shillingsburg, Peter, Scholarly Editing in the Computer Age. Theory and Practice, Ann Arbor, The University of Michigan Press, 1996.

Siemens, Ray, et al., «Toward Modeling the Social Edition: An Approach to Understanding the Electronic Scholarly Edition in the Context of New and Emerging Social Media», Literary and Linguistic Computing, 27 (2012), 445-461. 
Spence, Paul, «Teatro clásico y humanidades digitales: el cruce entre método, proceso y nuevas tecnologías», Teatro de palabras: revista sobre teatro áureo, 7 (2013), 9-38.

—, «Edición académica en la era digital: modelos, difusión y proceso de investigación", Anuario Lope de Vega: Texto, literatura, cultura, 20 (2014), 47-83.

Tanselle, G. Thomas, «Foreword», Electronic Textual Editing, eds. Lou Burnard, Katherine O'Brien O'Keeffe, John Unsworth, New York, The Modern Language Association of America, 2006, 1-6. 